\title{
El Sistema Nacional Integrado de Salud en Uruguay y los desafíos para la Atención Primaria
}

\author{
The Integrated National Healthcare System in Uruguay \\ and the challenges for Primary Healthcare
}

Ana Sollazzo ${ }^{1}$

Rosario Berterretche ${ }^{1}$
${ }^{1}$ Departamento de Medicina Preventiva y Social, Facultad de Medicina. Universidad de la República. Avda. Alfredo Navarro 3051, Piso 3. CP 11600 Montevideo Uruguay. anasollazzo@gmail.com

\begin{abstract}
The article examines the results of the overview of PHC (Primary Healthcare) in Uruguay in 2009, within the context of the multicentric study of PHC with a view to identifying possibilities of redefining PHC as a strategy to achieve universal healthcare systems, taking into account the healthcare system's segmentation and fragmentation. The methodology included document analysis, key informant interviews and triangulation of information sources. The results presented are based on the analytical model structured in five dimensions: delivery, financing, resources, and integrated and intersectoral PHC. At the macro level, one can observe the recent implementation of a sector reform through the creation of the Integrated National Health System (SNIS) that favors the development of the PHC from an integrative standpoint. Actions have been defined to overcome the segmentation of the system and the fragmentation of care. However, at an operational level, the segmentation that existed before the SNIS as well as the low levels of healthcare coordination have yet to be modified. This is related to management ability and organizational factors. The lack of adequate human resources for the implementation of the PHC strategy is identified as a relevant factor.
\end{abstract}

Key words Primary Health Care, Health policy, Organization of health services
Resumen El artículo aborda resultados del análisis panorámico de la Atención Primaria en Salud (APS) en Uruguay en Uruguay en 2009, en el marco de un estudio multicéntrico con objetivo de identificar posibilidades para reorientar la Atención Primaria en Salud (APS) como estrategia para alcanzar sistemas de salud universales, considerando la segmentación del sistema y la fragmentación en la provisión. La metodología incluyó análisis documental, entrevistas con informantes clave y triangulación de fuentes de información. Los resultados se presentan en base al modelo analítico construido en cinco dimensiones: conducción, financiamiento, recursos, integralidad e intersectorialidad de la APS. A nivel macro se observa la reciente reforma sectorial con creación de un Sistema Nacional Integrado de Salud (SNIS) que favorece el desarrollo de la APS desde una concepción integradora. Se han definido acciones que apuntan a superar la segmentación del sistema y la fragmentación de los cuidados. Sin embargo a nivel operativo la segmentación presente antes del SNIS, así como el bajo nivel de coordinación de los cuidados no se han modificado aún. Esto se relaciona con la capacidad de gestión y factores organizacionales. La falta de recursos humanos adecuados para la implementación de la estrategia de APS, se identifica como factor relevante.

Palabras clave Atención Primaria, Politicas de salud, Organización de servicios de salud 


\section{Introducción}

En este artículo se recogen los resultados del análisis panorámico de la APS en Uruguay para el año 2009, en el marco del estudio multicéntrico sobre APS desarrollado con Argentina, Brasil y Paraguay.

Uruguay es un pequeño país de $176.000 \mathrm{~km}^{2}$ que integra el cono sur de América, ubicándose entre Argentina y Brasil. Desde el punto de vista económico el país se encuentra en una fase de recuperación posterior al período de recesión del año 2000. En la Tabla 1 se muestran algunos indicadores económicos y socio demográficos del país.

En la situación sociodemográfica se destaca una baja tasa de natalidad y de mortalidad y una elevada esperanza de vida al nacer que condicionan un marcado envejecimiento de la población (Tabla 1)1. De acuerdo a datos de 2007 las dos principales causas de muerte se deben a enfermedades del aparato circulatorio (317,4 por 100.000 hab.) y tumores (230,6 por 100.000 hab.). Desde el punto de vista de los recursos humanos en salud se destaca para el año 2009 una razón de 42 médicos y 12 enfermeras profesionales por cada 10.000 habitantes (Tabla 1 ).

En Uruguay, hasta el año 2005, el Estado por intermedio del Ministerio de Salud Pública (MSP) y la Administración de Servicios de Salud del Estado (ASSE), tenía a su cargo la atención de la población de menores recursos, dejando en manos del sector privado la atención de los grupos de población con capacidad de pago.

Tabla 1. Indicadores socio demográficos y económicos seleccionados. Uruguay, 2007/8.

\begin{tabular}{lr}
\hline Indicador demográficos & \\
Población total & 3.329 .341 hab. \\
Proporción de población urbana & $92,5 \%$ \\
Proporción de población menor de 15 años & $22,0 \%$ \\
Proporción de población mayor de 65 años & $15,8 \%$ \\
Tasa de analfabetismo & $2,1 \%$ \\
Tasa bruta de natalidad x 1.000 habitantes & 14,9 \\
Tasa bruta de mortalidad x 1.000 habitantes & 9,6 \\
Esperanza de vida al nacer & 76,5 \\
Indicadores económicos & 7.300 \\
PIB per cápita anual en dólares & $21,7 \%$ \\
Tasa de pobreza (como \% de población) & $1,7 \%$ \\
Tasa de indigencia (como \% de población) & $7,7 \%$ \\
Gasto en salud como \% del PBI
\end{tabular}

Fuente: Instituto Nacional de Estadística (INE) Estimaciones y Proyecciones'.
En el subsector público, además de ASSE, existen otros prestadores como el Hospital de Clínicas de la Facultad de Medicina, Sanidad de las Fuerzas Armadas, Sanidad Policial, Banco de Previsión Social, Banco de Seguros del Estado y los servicios médicos de cada una de las 19 Intendencias Municipales del país. En el subsector privado las Instituciones de Asistencia Médica Colectiva (IAMC) son las principales efectoras del sistema cumpliendo hasta ese momento un doble rol de aseguradoras y prestadoras de servicios, siendo organizaciones sin fines de lucro, en su mayoría de propiedad cooperativa de grupos médicos. Existe además un sector de seguros privados que cubren al $5 \%$ de la población de mayores recursos.

Por otra parte el Fondo Nacional de Recursos (FNR), persona pública no estatal, a través de un mecanismo de reaseguro universal cubre procedimientos definidos como altamente especializados.

A partir de las elecciones presidenciales de 2004 en que por primera vez en la historia del país asume el gobierno una coalición de izquierda, el Encuentro Progresista - Frente Amplio, se comienza a implementar un proceso de "Reforma Sanitaria".

La reforma sanitaria se ubica en un contexto de transformaciones sociales, económicas y políticas, y se basa en cambios complementarios y progresivos del modelo de gestión, del modelo de financiamiento y del modelo de atención a la salud $^{2}$. Estos cambios se plasman a través de la promulgación de un conjunto de leyes y decretos dentro de los que se destaca la Ley 18.211 de diciembre de 2007 que crea el "Sistema Nacional Integrado de Salud (SNIS)" .

Con el SNIS se constituye un Seguro Nacional de Salud cuyos objetivos son entre otros el garantizar el acceso universal, brindar atención integral a la salud a toda la población, lograr niveles de accesibilidad y calidad homogéneas y lograr justicia distributiva en el financiamiento del gasto en salud que asegure la equidad, la solidaridad y la sustentabilidad del sistema. Este conjunto de principios y valores sobre los que se sustenta el SNIS constituyen los elementos de "cambio en el modelo de gestión del sistema"

Desde el punto de vista de los cambios en el "modelo de financiamiento" el Seguro se financia a través de un Fondo único público y obligatorio, el Fondo Nacional de Salud (FONASA), al que aportan el estado, las empresas y los hogares beneficiarios del SNIS, reuniendo todos los aportes de la seguridad social. Este fondo es adminis- 
trado centralmente por la Junta Nacional de Salud (JUNASA), organismo desconcentrado del Ministerio de Salud Pública en el que participan usuarios, trabajadores de la salud y prestadores. La JUNASA compra servicios a las instituciones prestadoras integrales tanto públicas como privadas a través de la firma de contratos de gestión con las mismas. Los usuarios aportan al fondo según ingreso y existe libre elección del prestador por parte de los usuarios. El prestador está obligado a brindar una cobertura integral a través de una canasta básica de prestaciones. Estas instituciones conforman el Sistema Integrado y reciben del FONASA una cápita ajustada por sexo y edad así como por metas asociadas al cumplimiento de objetivos prestacionales (Figura 1).

El principal prestador público, ASSE, que funcionaba en la órbita del MSP como organismo desconcentrado se descentraliza en 2007 a partir de la promulgación de la ley $18.161^{4}$. Simultáneamente se da la posibilidad a los usuarios del sistema de optar por ASSE como prestador integral, opción que hasta ese momento no existía.

La conformación de un sistema mixto público-privado de prestadores reconoce una fuerte presencia de instituciones de tipo mutual (IAMCs). En este sentido uno de los objetivos expresamente definido del sistema es la comple- mentación de servicios que posibilite una mayor racionalización en la utilización de los recursos.

Si bien la imagen objetivo del SNIS es la incorporación de toda la población, se han definido etapas para la incorporación progresiva de diferentes grupos de población que abarcan los funcionarios públicos sin cobertura, todos los trabajadores formales y sus hijos menores de 18 años, los jubilados con bajos ingresos y los nuevos jubilados, los cónyuges de los trabajadores, los profesionales, las cajas de auxilio, etc. Esto ha implicado un aumento de la cobertura integral progresiva, desde diciembre de 2007 esperando para el año 2015 poder brindar una cobertura por FONASA al $80 \%$ de la población del país².

Los cambios en el modelo de gestión y de financiamiento se consideran instrumentos para el logro de un nuevo "modelo de atención". En este sentido la propuesta de reforma retoma el documento de la Organización Panamericana de la Salud (OPS), la Renovación de la Atención Primaria de Salud de las Américas, en la que se propone la construcción de Sistemas de Salud basados en APS. Esta se enfoca sobre el conjunto del sistema de salud como una estrategia de organización.

Desde esta perspectiva el objetivo del estudio es identificar límites y posibilidades para reorientar la Atención Primaria en Salud como estrate-

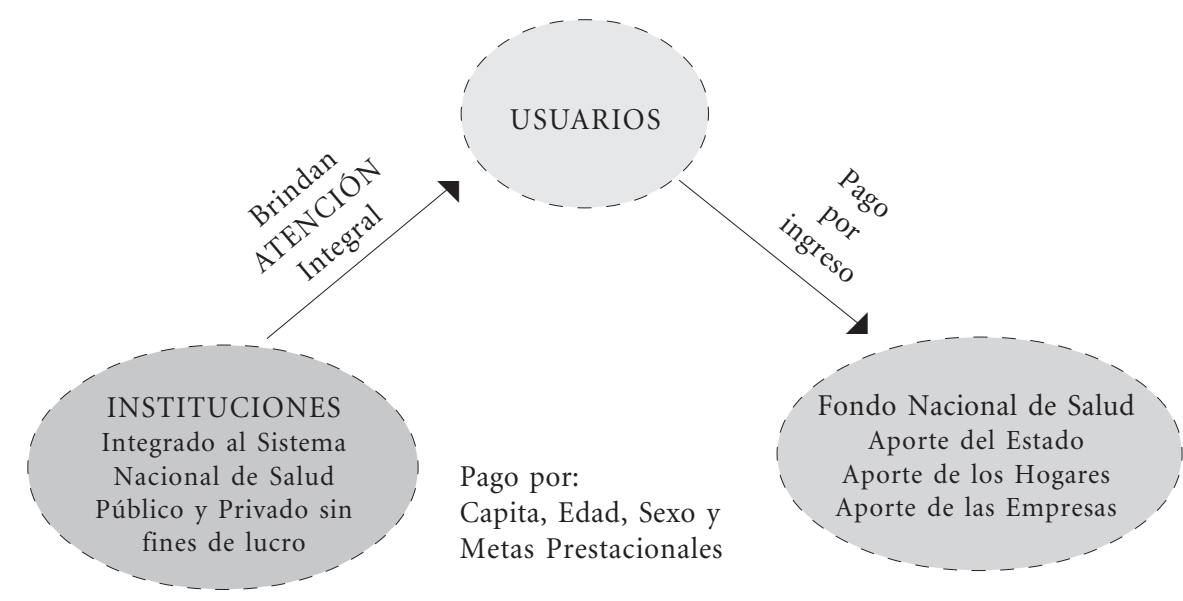

Figura 1. La conformación del Seguro Nacional de Salud en Uruguay. 
gia para alcanzar sistemas de salud integrados y universales, considerando el grado de segmentación del sistema de salud y la fragmentación en la provisión de los servicios.

\section{Metodología}

Se trata de una investigación multicéntrica, cualitativa, basada en entrevistas a informantes clave, trianguladas con la revisión y análisis documental y de estadísticas.

Las categorías de informantes clave entrevistados fueron: gestores, expertos, entidades profesionales y organizaciones de la sociedad civil. Se seleccionaron entrevistados que ocuparan puestos de conducción en las organizaciones de referencia y en el caso de la categoría expertos, se consideró además, personas reconocidas en el medio como tales. Para el análisis de las entrevistas se procedió a realizar un análisis de contenido según las diferentes dimensiones y variables de la matriz del estudio común a los cuatro países, capacidad de conducción de la APS, financiamiento, recursos, integralidad y continuidad de los cuidados, intersectorialidad y futuro de la APS (Cuadro 1).

Se realizaron 33 entrevistas (17 Gestores, 8 expertos, 4 representantes de la Sociedad Civil Organizada y 4 entidades profesionales). Por otra parte se realizó una búsqueda bibliográfica consultando las bases Scielo y Lilacs.

\section{Resultados}

\section{Dimensión I - \\ Capacidad de conducción de la APS}

En relación al cambio del modelo de atención se concibe a la "APS como una estrategia" para la reorganización del sistema de salud ${ }^{5}$. Esto queda plasmado en la Ley de creación del SNIS bajo el título de "Red de Atención en Salud" en la que se define la organización del sistema de atención a la salud: se organizará en redes por niveles de atención según las necesidades de los usuarios y la complejidad de las prestaciones. Tendrá como estrategia la atención primaria en salud y priorizará el primer nivel de atención $n^{3}$.

Cuadro 1. Dimensiones y subdimensiones del estudio de APS.

\begin{tabular}{|c|c|c|}
\hline $\mathbf{F}$ & Dimensiones & Subdimensiones \\
\hline $\begin{array}{l}\text { A } \\
\text { G } \\
\text { M } \\
\text { E }\end{array}$ & 1. Capacidad de conducción de la APS & $\begin{array}{l}\text { Políticas relacionadas con la APS } \\
\text { Implementación de políticas de APS } \\
\text { Instituciones responsables por la provisión de APS } \\
\text { Actores involucrados en la APS }\end{array}$ \\
\hline $\begin{array}{l}\mathrm{T} \\
\mathrm{A} \\
\mathrm{C}\end{array}$ & 2. Financiamiento de la APS & $\begin{array}{l}\text { Fuentes de financiamiento y gasto en APS } \\
\text { Modalidad de asignación de recursos para la APS } \\
\text { Sistemas de pago a los recursos humanos en la APS }\end{array}$ \\
\hline $\begin{array}{l}\text { Ó } \\
\text { N }\end{array}$ & $\begin{array}{l}\text { 3. Provisión, recursos humanos e } \\
\text { integralidad de la APS }\end{array}$ & $\begin{array}{l}\text { Tipo de institución prestadora de APS } \\
\text { Tipo de servicio de salud que proveen APS y acceso } \\
\text { Organización del trabajo en APS } \\
\text { Cuantificación y cualificación de los recursos humanos }\end{array}$ \\
\hline $\begin{array}{l}\text { S } \\
\mathrm{E} \\
\mathrm{G} \\
\mathrm{M}\end{array}$ & $\begin{array}{l}\text { 4. Integración de la APS en el Sistema } \\
\text { de Salud y continuidad del cuidado }\end{array}$ & $\begin{array}{l}\text { Continuidad de la atención } \\
\text { Longitudinalidad de la atención } \\
\text { Integración vertical y articulación horizontal de la APS }\end{array}$ \\
\hline $\begin{array}{c}\mathrm{E} \\
\mathrm{N} \\
\mathrm{T} \\
\mathrm{A} \\
\mathrm{C} \\
\mathrm{I} \\
\text { Ó } \\
\mathrm{N}\end{array}$ & $\begin{array}{l}\text { 5. Articulación intersectorial y relaciones } \\
\text { de la APS con actores con actores } \\
\text { sociales y políticos }\end{array}$ & $\begin{array}{l}\text { Relación de la APS con los diferentes niveles de } \\
\text { gestión del sistema } \\
\text { Relación de la APS con los diferentes sectores del } \\
\text { gobierno } \\
\text { Relación de la APS con diferentes actores de la } \\
\text { sociedad civil }\end{array}$ \\
\hline
\end{tabular}


Desde el punto de vista de los entrevistados también se entiende la APS como una estrategia de carácter político que orienta y conduce toda la atención a la salud del país. Sin embargo pueden identificarse algunas tensiones entre los diferentes actores. En una amplia escala de matices se podría describir los extremos de la tensión planteada entre los actores que ponen el énfasis en los aspectos de promoción y prevención vinculados a la salud por sobre todos los otros aspectos, incluso dejando expresamente de lado el nivel asistencial y en el otro extremo en el que se sostiene la necesidad de abordar la APS desde el punto de partida de la buena resolución de los problemas de salud - enfermedad desde una perspectiva clínica, lo que permite abordar con legitimidad los aspectos promocionales y preventivos.

En relación a la relación APS y el Primer Nivel de Atención algunos entrevistados plantean que existe una "dificultad en entender el concepto". Existen concepciones en donde se equipara la APS con el de primer nivel de atención y menciona: $E n$ el país hoy ya nadie considera APS igual a primer nivel de atención al menos en ámbitos académicos $y$ de decisión. Estoy segura que desde lo político queda mucho camino por andar con respecto a esto.

A través de la reforma se enfatiza en la capacidad de "rectoría" de la autoridad sanitaria y dentro de ella su capacidad de "conducción" entendida como la capacidad del MSP de formular, organizar y dirigir la ejecución de la política nacional de salud ${ }^{6}$. En este sentido se han promulgado un conjunto de leyes que materializan la reforma. Un hito importante del proceso es la Ley de descentralización de ASSE. Antes de su promulgación el MSP tenía un doble rol de control y prestación que lo convertía en "juez y parte" del sistema por lo que desarrolló un escaso control, en particular del sector público.

En cuanto a la "participación en la formulación de políticas", se crea en 2005 el Consejo Consultivo para la Implementación del Sistema $\mathrm{Na}$ cional Integrado de Salud, convocado por el Poder Ejecutivo. Se contó con una amplia participación de representantes de diversas organizaciones del sector con el objetivo de consensuar los lineamientos de la propuesta de reforma. Sin embargo ninguno de los actores entrevistados identificó esta instancia como un ámbito de participación en la formulación de las políticas de salud.

En relación a la "operacionalización" de las políticas de APS, se han diseñado un conjunto de instrumentos que favorecen su implantación. Estos elementos son bien identificados por parte de los actores. Sin embargo se mencionan difi- cultades para su concreción, se identifica que el avance teórico y el nivel de discusión del tema no se acompasan con una práctica acorde. Se hace énfasis en la dificultad de la evaluación y el control de las acciones que logren una retroalimentación que permita a su vez mejorar las prácticas.

\section{Dimensión II - Financiamiento de la APS}

Desde el punto de vista del "gasto y del financiamiento" el país destinaba cerca de un $9 \%$ de su PBI al sector salud. Del total del gasto el 50\% provenía de fuentes públicas. Sin embargo solo el $25 \%$ del total del gasto se destinaba a los proveedores públicos según el último estudio de Cuentas Nacionales de Salud de 2004. Los aportes del bolsillo alcanzaban un $18 \%$ de las fuentes de financiación privada ${ }^{7}$.

Con la creación del SNIS se redefinen los mecanismos de financiamiento de la atención a la salud a través de la creación de un Seguro Nacional de Salud que funciona bajo el esquema de seguro social, los individuos aportan según su capacidad contributiva y reciben atención según sus necesidades de salud. Se apunta a la equidad de la financiación, a través de una contribución progresiva. La asignación de recursos a los prestadores se realiza a través de transferencias globales en base a un valor per cápita o "cuota salud" ajustadas por riesgo (edad y sexo) y al cumplimiento de metas asistenciales. Las metas se plantean como incentivos en relación a programas específicos vinculados al fortalecimiento de las acciones de APS. Desde el punto de vista de la afiliación han ingresado al FONASA el 43,9\% de la población, mientras que en el $56,1 \%$ restante la cobertura es financiada por otros mecanismos.

En cuanto al financiamiento de la APS, no existen recursos asignados de forma específica a la misma. En el sector público se realizó una estimación del porcentaje del gasto en APS que lo ubica en el 22\% del total del gasto del año $2008^{8}$.

En este sentido los entrevistados refieren que hay una discrepancia en la normativa y su correlato desde el punto de vista de los recursos asignados, si bien desconocen el porcentaje de gasto en APS se considera que los recursos asignados son extremadamente insuficientes.

\section{Dimensión III - \\ Provisión y recursos para la APS}

Desde el punto de vista de "la provisión" el sistema de salud uruguayo se encuentra fuertemente segmentado. Si observamos la oferta de 
servicios aparecen "ASSE y las IAMCs", como los principales prestadores integrales públicos y privados respectivamente, junto a otros múltiples efectores que ofrecen prestaciones integrales o parciales.

ASSE cuenta en Montevideo con una Red de Atención del Primer Nivel (RAP) que recibe y ejecuta presupuesto propio a través de 12 Centros de Salud, 32 Policlínicas y 70 Consultorios de Área9 . En el interior no existe una organización específica de servicios de APS, ésta se brinda en ASSE a través de centros de salud, policlínicas y consultorios dependientes de los hospitales departamentales. En las IAMCs, los servicios de APS corresponden a los servicios ambulatorios de las 41 IAMCs distribuidas en todo el territorio.

A partir de la reforma se accede a la cobertura integral a través de la Seguridad Social. En 2009 el Registro Único de Cobertura de Asistencia Formal (RUCAF) registraba una cobertura formal del $95 \%$ del total de la población. En relación al tipo de prestador integral el $37,1 \%$ de la población se adscribía a ASSE, el 55,3\% a las IAMCs, el $2,3 \%$ a los seguros integrales, el 3,5\% a Sanidad Militar y $1,7 \%$ a Sanidad Policial.

Los prestadores integrales del SNIS están obligados a ofrecer a sus beneficiarios un Programa Integral de prestaciones que es definido taxativamente por la JUNASA ${ }^{10}$. Esta obligación queda formalizada a través de los Contratos de Gestión que se firman entre ésta y los prestadores integrales. En relación a estos programas los actores entrevistados coinciden en señalar el poco desarrollo de los mismos a nivel de la rehabilitación y los cuidados paliativos.

Por otra parte y en lo que hace a la segmentación del sistema la JUNASA define una política de coordinación y complementación asistencial entre prestadores que permita un uso más intensivo y más eficiente de las capacidades existentes. En 2008 se relevaron 80 convenios, que involucraron por lo menos 45 organizaciones diferentes.

Desde el punto de vista de la accesibilidad económica la existencia de copagos en el sector de las IAMCs para prestaciones ambulatorias y del primer nivel de atención, se identifican como una barrera al acceso. En este sentido la actual política de regulación definió la exoneración y/o la disminución del monto de los mismos para los pacientes diabéticos, hipertensos, realización de controles de salud de la mujer y la mujer embarazada y controles de salud de niños y adolescentes.

En ASSE si bien no existen copagos, existen en el primer nivel de atención barreras al acceso desde una perspectiva organizacional que se ha- cen críticas en lo que refiere al acceso a los medicamentos y exámenes de laboratorio. La RAP en Montevideo ha dispuesto una serie de medidas tendientes a mejorar la accesibilidad a estas prestaciones?.

Sin embargo, los actores entrevistados plantean que hay sectores importantes de la población a los cuales el sistema de salud no llega ${ }^{10}$.

La JUNASA también ha normatizado en lo referente a los tiempos de espera en el primer nivel de atención para el acceso a las especialidades básicas (obtención de una consulta en un tiempo máximo de 24 hs. para medicina, pediatría y ginecología) y también para los otros niveles de atención y la cirugía ${ }^{11}$. Las primeras evaluaciones de estos estándares se han realizado para el sector de las IAMCs, donde se observa un incumplimiento en los tiempos de espera en el primer nivel de atención estipulados; en ginecología es de 3 a 4 días y en pediatría de 1 a 2 días $^{12}$. Aún no se cuenta con información disponible a nivel del sector público.

En relación a los "recursos humanos" encontramos en el país una disponibilidad de médicos de 4,1 cada mil habitantes en el año 2005. Su distribución geográfica es dispar con una mayor concentración a nivel de la capital: $73 \%$ del to$\mathrm{tal}^{13}$. Se constata un alto grado de especialización con una distribución al interior de las diferentes especialidades muy heterogénea y un alto grado de multiempleo.

En relación al personal de enfermería encontramos para el mismo año una relación de 0,3 enfermera por médico, relación que viene en descenso $^{13}$.

Se constata un descenso de las especialidades médicas generalistas y del personal de enfermería, recursos imprescindibles para el desarrollo de la APS en el marco del cambio en el modelo de atención.

En cuanto a la disponibilidad de recursos humanos por subsector, para 2005 el sector privado disponía del doble de médicos por mil usuarios que ASSE y cerca de un 50\% más de enfermeros y técnicos no médicos. Sin embargo, la relación de recursos médicos y no médicos cada 1000 usuarios, ha mejorado de forma sustancial en ASSE si comparamos el año 2008 con el 2005, pasando la relación de médicos cada mil usuarios de 3,8 a 4,7 y la de enfermeros cada mil usuarios de 4,77 a $5,20^{8}$.

En el subsector privado el régimen de trabajo establecido determina una baja dedicación horaria de los cargos y diferencias salariales entre categorías laborales. El médico general tiene una 
remuneración base y los salarios aumentan en relación al grado de especialización tanto en su componente fijo como variable, con una mayor retribución para las especialidades que se desempeñan en los niveles de mayor complejidad asistencial, del segundo y tercer nivel de atención.

En el caso de ASSE, el nivel salarial al 2005 es menor que el de las IAMC. El sistema de remuneración se corresponde en términos generales con un salario fijo, contratos de alta dedicación horaria y una menor diferenciación salarial entre especialidades que en el sector privado. A partir del año 2005 se han instalado progresivamente Equipos de Área y se integraron los médicos de familia a la red de efectores de primer nivel. La brecha salarial existente en relación al sector privado ha disminuido.

Los entrevistados refieren que los efectores privados tienen una forma de organización del trabajo totalmente contraproducente con la orientación de APS (por ejemplo pago por paciente), mientras que los efectores públicos favorecen el desarrollo de la estrategia de APS (contratación de médicos de área, estabilidad laboral, horario de 36 hs semanales, etc.).

En cuanto a la conformación del equipo básico de Atención Primaria los entrevistadeos plantean posiciones diferentes. Algunos refirieron que no se puede definir de antemano, sino que depende de las características demoepidemiológicas, culturales, socioeconómicas, organización sanitaria, etc. En el otro extremo se refiere que el equipo básico debería estar conformado por un Medico de Medicina Familiar y Comunitaria y una licenciada en enfermería; existiendo otras posiciones intermedias. Sí hay acuerdo en que el Equipo debe poder resolver el 80 a $90 \%$ de las necesidades de salud de la población y que la capacitación para trabajar en el primer nivel de atención es muy específica.

En cuanto a la formación de los recursos humanos para trabajar en el primer nivel de atención, los entrevistados refirieron que los planes de estudio de la carrera de medicina no se adecuaban a las necesidades del país, no se preparan médicos para trabajar en el primer nivel de atención ni en una estrategia de APS. Se destaca como hecho positivo que el nuevo plan de estudios toma como base filosófica elementos fundamentales de la estrategia de la Atención Primaria de Salud y la creación de la especialidad de Medicina Familiar y Comunitaria ${ }^{14}$.

\section{Dimensión IV - Integralidad y continuidad}

En relación a la "integralidad y continuidad" de la atención la legislación vigente define 11 programas prioritarios de salud, así como un catálogo de prestaciones obligatorias. Los programas son controlados en su ejecución por la JUNASA y actualizados periódicamente por el MSP. Incluyen actividades de promoción, protección, recuperación, rehabilitación y cuidados paliativos de las personas, incluyendo el acceso a la tecnología y medicamentos aprobados en el Formulario Terapéutico de Medicamentos.

Desde el punto de vista de los "entrevistados se refiere que la cartera de servicios del primer nivel" es inadecuada y que existen diferencias geográficas importantes. En Montevideo existe una sobreoferta de servicios con duplicaciones y no solo entre distintos prestadores públicos o privados, sino incluso dentro de las mismas instituciones. También se constatan diferencias entre el medio urbano o rural. Se plantea la necesidad de una regionalización que implique la coordinación y racionalización de los recursos. Por otra parte se mencionan dificultades a nivel de la implementación de la rehabilitación y los cuidados paliativos.

En relación a la integración vertical de la APS el SNIS se organiza por niveles de atención y la JUNASA es quien establece y debe asegurar los mecanismos de referencia y contrareferencia entre los distintos niveles en base a la normativa del MSP.

En general los diferentes entrevistados coinciden en que, salvo excepciones, la integración vertical es insatisfactoria y hay serias dificultades para lograr una articulación con los demás niveles. Asimismo se plantea que la integración depende de la región (interior o capital), de los centros y muchas veces de la voluntad de los gestores. Por otra parte se plantea que la integración y la resolutividad es alta cuando se necesita asistencia inmediata pero cuando no es urgente falla, por ejemplo en la coordinación de estudios, interconsultas con especialistas, etc.

Los entrevistados refieren que los mecanismos de referencia y contrarreferencia son un problema, o que directamente no existen, lo que es una gran debilidad del sistema.

En relación a la articulación horizontal el SNIS obliga a los prestadores a brindar atención integral tomando como marco los contenidos de los Programas. En este sentido se han definido para los Programas prioritarios un conjunto de 
guías clínicas que están disponibles. En particular en lo que refiere a las enfermedades crónicas no trasmisibles el MSP desarrolla un programa piloto para el cambio de modelo de atención a la salud el "Programa Previniendo", con financiamiento del Banco Mundial. Su propósito es reducir la morbimortalidad de las enfermedades no trasmisibles prevalentes (obesidad/sobrepeso, hipertensión, diabetes y cáncer de colon) y homogeneizar la práctica clínica en el Primer Nivel de Atención, para realizar la detección precoz y control adecuado de estas patologías.

Desde el punto de vista de los entrevistados se considera que el grado de integración de la promoción, prevención y tratamiento es razonable, pero la rehabilitación es catalogada como insatisfactoria.

En relación al vínculo el SNIS ha incorporado dentro de las metas prestacionales la asignación de un médico de referencia a sus beneficiarios. Esta meta apunta al seguimiento longitudinal de los usuarios dentro del sistema y es de reciente implementación.

Desde el punto de vista de los entrevistados se plantea que es necesario generar el compromiso de los trabajadores de la salud ya que hasta que no se comprometan y se convenzan de la necesidad de este tipo de atención no será posible brindar una atención de primer nivel con una estrategia de APS, se debe trabajar por lo que un entrevistado llamó "humanización de los lugares de trabajo".

Se mencionan como experiencias exitosas en relación a la integralidad y continuidad del cuidado el programa aduana y los espacios adolescentes.

\section{Dimensión V - Articulación intersectorial y relaciones de la APS con actores sociales y políticos}

En cuanto a la articulación intersectorial, en el año 2005, se crea el Gabinete Social, el cual está integrado por los titulares de los Ministerios de Economía y Finanzas, de Educación y Cultura, de Trabajo y Seguridad Social, de Salud Pública, de Vivienda, Ordenamiento Territorial y Medio Ambiente y de Desarrollo Social, quien lo preside. Sus cometidos son entre otros, establecer estrategias y lineamientos de acción conjunta en materia de Políticas Públicas Sociales, articular atribuciones y campos de actuación de naturaleza intergubernamental entre las instancias nacionales y municipales y de carácter sectorial con los diferentes órganos y entes públicos.
Se describieron múltiples experiencias de articulación intersectorial, entre las que podemos mencionar en Montevideo y desde la RAP, la organización de Mesas de Articulación social Regional y proyectos interinstitucionales para mejorar la salud materno infantil; en el interior del país, la instalación de un Plan de Desarrollo de la Atención Primaria de Salud (DAPS) en Tacuarembó, en los Departamentos de Florida y Artigas el desarrollo de una experiencia de Sistema Local de Salud. En Cerro Largo, las acciones desarrolladas por la Fundación Quebracho - Promoción en Comunidades Rurales quienes diseñaron un modelo de desarrollo local. Un entrevistado hizo referencia a la existencia en este departamento del Comité Departamental de Salud, que funciona de forma ininterrumpida desde el año 1985 hasta el momento actual.

Asimismo, uno de los principios rectores de la acción del MSP ha sido el de alentar la participación social en los procesos de formación de las políticas de salud. Ejemplo de esto los Consejos Consultivos, JUNASA como órgano de administración del Seguro Nacional de Salud con la participación de un representante de los usuarios, uno de los trabajadores y otro de las empresas del sector salud, etc.

En ASSE se impulsaron las Asambleas Representativas de Usuarios en las diferentes Zonas de Salud de Montevideo y se propusieron e implantaron a lo largo de dos años las Comisiones de participación en los 12 Centros de Salud, ámbitos tripartitos que incluyen a los equipos de gestión, representantes de los trabajadores y de los usuarios organizados, a efectos de monitorear el desarrollo de los programas y las debilidades y fortalezas del proceso de cambio de modelo de atención.

Para los entrevistados esta Administración ha actuado como un facilitador de la articulación intersectorial. Sin embargo y a pesar de existir experiencias exitosas, la mayoría de los actores mencionan una articulación programada pero que no ha sido sistemática ni es homogénea en todo el país.

Finalmente los entrevistados identifican como un "factor facilitador para la implementación de la estrategia de APS" el que se pudiera contar con recursos humanos comprometidos, bien remunerados, valorizados y capacitados. A nivel de los "factores obstaculizadores" se identifican los intereses sectoriales, a veces de las diferentes instituciones, a veces marcadas por lo económico, pero a veces por espacios de poder, la cultura, la idiosincrasia uruguaya, una actitud conservado- 
ra y pasiva. Los actores refieren que los resultados de los cambios implementados se podrán observar a mediano y largo plazo y que el desafío es el cambio cultural que se deberá gestar en la población, en las instituciones, en los técnicos, e identifican a los médicos como el componente de mayor resistencia.

La "visión a futuro" es optimista, supeditada fundamentalmente a la continuidad de las orientaciones y compromisos políticos asumidos y a la capacidad de documentar y evidenciar resultados y el empoderamiento de las personas acerca de los resultados de la implementación de una estrategia de APS.

\section{Discusión y conclusiones}

El proceso de reforma iniciado hace énfasis en la capacidad de "conducción" del MSP con la creación del SNIS que integra prestadores públicos y privados. Se crea un seguro de Salud obligatorio con contratación de prestadores, vinculado a la condición laboral. A través de la JUNASA se realizan contratos explícitos con los proveedores donde se establecen el nivel y el tipo de prestaciones. Uno de los ejes de la reforma es el cambio del modelo de atención que se centra en la APS entendida como una estrategia para la reorganización del sistema de salud.

Si bien los entrevistados comparten esta concepción aparecen tensiones entre quienes enfatizan los aspectos promocionales y preventivos y quienes dan peso a los aspectos clínicos que dan la legitimidad para un abordaje integral.

En relación a la operacionalización de las políticas se han generado mecanismos que favorecen su implantación tal como son los Programas Integrales de prestaciones y las metas asistenciales. Sin embargo debemos tener en cuenta que tal como es expresado por varios autores un problema común es la idea de que las políticas enunciadas por ley o descritas en documentos formales del gobierno, serán inmediatamente implementadas. Por diversas razones los resultados de las reformas pueden diferir bastante de las intenciones y enunciados originales de las políticas ${ }^{15}$. En este sentido los entrevistados reconocen que se ha alcanzado un muy buen nivel de discusión y conceptualización pero se plantea que éste no se condice con las prácticas y se reconocen dificultades de en la coordinación público-privada, sobre todo a nivel de la capital. A nivel público se reconoce un mayor grado de avance en la capital. Esto acuerda con la afirmación realizada por Björ- kman el sistema público tiene muchas fallas pero también tiene muchas cualidades ${ }^{15}$.

En relación al "financiamiento" el seguro se financia mediante contribuciones progresivas que se vuelcan a un fondo común (FONASA). Se financia a los proveedores por un per cápita ajustado por riesgo (edad y sexo) e incentivos según desempeño en el cumplimiento de metas prestacionales que se dirigen a fomentar acciones que tienden al desarrollo y fortalecimiento de la APS. Sin embargo no existe en general autonomía en el manejo del presupuesto asignado a la APS.

Desde el punto de vista de la "provisión y los recursos para la APS" existe una cobertura universal, a través de un sistema segmentado con múltiples prestadores públicos y privados. La población que tiene derechos a recibir prestaciones integrales por estar adscripta a un servicio de salud público o privado independientemente de quien financie la afiliación (cobertura formal) asciende al 95\%.

La cobertura es integral y de libre elección, y está asegurada a través de la obligatoriedad de los prestadores integrales de brindar un Programa Integral de Prestaciones a través de contratos de gestión con la JUNASA. Desde la perspectiva de los entrevistados se considera que la accesibilidad geográfica no es adecuada y que "los servicios no llegan a nivel rural”. En este sentido el Grupo de Consenso sobre un Marco de Evaluación de la Atención Primaria en América Latina plantea como uno de los factores condicionantes para el logro de la APS la necesidad de una asignación y distribución de recursos basadas en las necesidades de la población ${ }^{16}$. Sin embargo, este hecho no se ha constatado en la presente investigación.

Desde el punto de vista de la accesibilidad se han normatizado los tiempos de espera para la consulta en el primer nivel de atención. Una evaluación del sector privado observó tiempos de espera superiores a la norma (24 horas desde el momento de la solicitud) en relación a la consulta con pediatra (entre 1 y 2 días) y ginecólogo (entre 3 y 4 días).

En relación a los recursos humanos se constata que existe un elevado número de médicos y una baja disponibilidad de enfermeras concentrándose los primeros en Montevideo y en el sector privado. Poseen un alto grado de especialización, coexistiendo un elevado multiempleo así cómo diferentes formas de contratación y remuneración según sector en el que desempeñan. Estas características se han identificado como factores limitantes de las reformas tal como lo plantea Björkman ${ }^{15}$ en "Reformas de salud en pers- 
pectiva comparada”. En el análisis planteado por este autor la cantidad de recursos humanos calificados y motivados disponibles, así como la cultura organizacional son una de las barreras más importantes de las reformas sanitarias. Dicho autor identifica como otra barrera la falta de incentivos para los profesionales ${ }^{15}$.

Desde el punto de vista de la conformación de los equipos de trabajo en APS no existe aún normativa, pero desde el punto de vista de los entrevistados se plantea una amplia gama de opciones que tienen en común el médico de familia y la enfermera.

Desde el punto de vista de la formación de los recursos humanos no se identifica una política explícita y los entrevistados consideran que no hay una adecuación de la formación para dar respuesta a las necesidades del país.

Desde el punto de vista de la "integralidad y continuidad", entendidas como la articulación de las acciones preventivas, curativas de rehabilitación y paliativas en todos los niveles de compleji$\mathrm{dad}^{17}$, si bien se definen programas integrales $y$ un catálogo de prestaciones obligatorias los "entrevistados refieren que la cartera de servicios del primer nivel" es inadecuada y que existen diferencias geográficas importantes, con una sobreoferta de servicios y duplicaciones entre prestadores públicos o privados e incluso dentro de las mismas instituciones. También se constatan diferencias entre el medio urbano o rural. Se plantea la necesidad de la regionalización que implique la coordinación y racionalización de los recursos. En este sentido la mala coordinación entre diferentes actores es reconocida por Björkman como una señal de poca capacidad de implementación de las reformas ${ }^{15}$.

En relación a la integración vertical del cuidado se define un sistema de atención organizado por niveles.

Desde la perspectiva de los entrevistados se coincide en que la integración es insatisfactoria hay serias dificultades para lograr una articulación de la APS con los demás niveles, con la mayor debilidad en la referencia -contrarreferencia.

A nivel de la integración horizontal los programas incluyen la atención integral. En este sentido se percibe como un déficit la rehabilitación y los cuidados paliativos.

En relación a la atención longitudinalidad de la atención se ha incorporado en las metas prestacionales la adscripción de la población a un médico de referencia. Sin embargo por parte de los entrevistados se plantea que hay un rezago en su concreción. Se plantean como experiencia exi- tosa el "Plan Aduana" de seguimiento y control de los recién nacidos.

Según Almeida et al. ${ }^{18}$, las principales estrategias para la integración de los sistemas de salud están relacionadas con la territorialización de los servicios y la adscripción de población, la informatización de historias clínicas, la centralización de la coordinación de consultas, equipos de especialistas como apoyo para la Atención Primaria de Salud y la creación de redes como gestión regional entre otras. En este sentido en Uruguay tal como se describió anteriormente dichos instrumentos poseen un muy escaso desarrollo.

En relación a la "articulación intersectorial" se identifica la creación del Gabinete Social que es percibido por los entrevistados como un facilitador de la articulación intersectorial. Se identifican por parte de los entrevistados múltiples experiencias de articulación intersectorial con la sociedad civil en todo el país, pero se menciona que las mismas no son homogéneas.

Podemos concluir que las opiniones de los diversos actores presentaron cierto grado de complementación, desde un nivel más técnico, político o vivencial, dependiendo del actor considerado. Solo se identificaron tensiones en dos aspectos: "conceptualización de APS y conformación del equipo básico de APS".

A "nivel macro" se han implementado a partir de 2007 una serie de cambios que favorecen el desarrollo de una APS con una "concepción" abarcadora, como una forma de "reorganización del sistema de salud y de cambio del modelo de atención".

Se han definido acciones que apuntan a superar la "segmentación del sistema y la fragmentación de los cuidados" a través de los cambios en el financiamiento, el derecho a la cobertura, el acceso, etc.

Sin embargo en un nivel "operativo" la segmentación presente antes de la creación del Sistema Nacional Integrado de Salud, así como el bajo nivel de coordinación de los cuidados nos se han modificado. Estos factores están de alguna manera relacionados a las "capacidades de gestión a nivel institucional y con factores organizacionales", teniendo en opinión de los entrevistados un gran peso la cultura organizacional.

La no existencia en el país de "recursos humanos" en cantidad ni cualiltativamente preparados para responder a esta estrategia es una debilidad importante.

Una vez creadas las condiciones a nivel macro social nos encontramos en el desafío de actuar sobre la integración de los servicios y la continuidad del cuidado. 


\section{Colaboradores}

A Sollazzo y R Berterretche participaron del diseño metodológico, revisión documental, realización de entrevistas, análisis, triangulación y sistematización de los resultados, así como de la redacción del documento final, construyendo de manera conjunta la discusión y conclusiones, contando con la colaboración de las Dras. Delia Sánchez, Estela Nogueira y Ana Kuster en distintas etapas del desarrollo de la investigación.

\section{Agradecimientos}

A la Red de Investigación en Sistemas y Servicios de Salud del Cono Sur por el apoyo y al International Development Research Centre (IDRC) por la financiación de la investigación.

\section{Referencias}

1. Instituto Nacional de Estadísticas Uruguay. Indicadores demográficos. [accedido 2009 abril 6]. http://www.ine.gub.uy/socio-demograficos/ demograficos2008.asp

2. Olesker D, González T. La construcción del Sistema Nacional Integrado de Salud (2005-2009). Montevideo, Uruguay: Ministerio de Salud Pública; 2009.

3. Ley de SNIS n ${ }^{\circ} 18.211$ del 5 de diciembre de 2007. Ley de Sistema Nacional Integrado de Salud. Poder Legislativo. Uruguay. [accedido 2009 setiembre 20]. Disponible en: http://www.parlamento.gub.uy

4. Ley de descentralización de ASSE nº 18.161 de 2007. Ley de descentralización de Administración de Servicios de Salud del Estado. Uruguay: Poder Legislativo [accedido 2009 setiembre 20]. Disponible en: http://www.parlamento.gub.uy.

5. Uruguay. Ministerio de Salud Pública. Dossier Nacional de Atención Primaria en Salud y la integración con otros niveles de atención. Montevideo, Uruguay: Programa Eurosocial Salud; 2007. Intercambio III. (No publicado).

6. Organización Panamericana de la Salud. La función de rectoría en salud y el fortalecimiento institucional de las autoridades sanitarias nacionales y subnacionales. En: Organización Panamericana de la Salud. La salud Pública en las Américas. [accedido 2009 octubre 10]. Disponible en: http://www. campusvirtualsp.org.

7. Uruguay. Ministerio de Salud Pública. Cuentas nacionales en salud 2004, Uruguay. [accedido 2009 setiembre 20]. Disponible en: www.msp.gub.uy.

8. Isabella F, Katzkowikcz N, Yapor M. Documento de trabajo de la Dirección de Planificación Estratégica de ASSE. Uruguay: Doc. Int. Uruguay; 2009.

9. Benia W. Jornadas de Atención Primaria de Salud de la Red de Atención del Primer Nivel. "Creciendo en salud; 2009; RAP-ASSE. Montevideo; 2009.

10. Uruguay. Ministerio de Salud Pública. Programa Integral de Salud y Catálogo de Prestaciones. Uruguay: Ministerio de Salud Pública; 2008. Anexo I y II.

11. Uruguay. Ministerio de Salud Pública. Derechos de los usuarios. Junta Nacional de Salud, Uruguay 2008. [accedido 2009 setiembre 18]. Disponible en: http:/ /www.msp.gub.uy/ucjunasa_3061_1.html.

12. Uruguay. Ministerio de Salud Pública. Descripción $y$ análisis de indicadores asistenciales del subsector privado de salud, 2007-2008. Uruguay: División Economía de la Salud, Área Asistencial del MSP. Documento int. Uruguay; 2009.

13. Organización Panamericana de la Salud(OPAS). Perfil de recursos humanos del sector salud en Uruguay. Montevideo: OPAS; 2007.

14. Uruguay. Facultad de Medicina. Plan de Estudios Carrera de Doctor en Medicina. Montevideo, Uruguay: Asamblea del Claustro, Facultad de Medicina, Universidad de la República (UDELAR); 2007.

15. Björkman JW. Reformas de saúde em perspectiva comparada - uma questão sem fim .... ou uma busca improvável. Cien Saude Colet 2009; 14(3):763-770.

16. Haggerty JL, Yavich N, Báscolo EP, Grupo de Consenso sobre un Marco de Evaluación de la Atención Primaria en América Latina. Un marco de evaluación de la atención primaria de salud en América Latina. Rev Panam Salud Publica 2009; 26(5):377-384. 
17. Almeida PF, Giovanella L, Mendonça MHM, Escorel S. Notas para el estudio de la Atención Primaria en contextos de sistemas de salud segmentados. Rev Salud Publica 2010; 12(Supl.1):77-880.

18. Almeida PF, Giovanella L, Mendonça MHM, Escorel S. Desafios à coordenação dos cuidados em saúde: estratégias de integração entre níveis assistenciais em grandes centros urbanos. Cad Saude Publica 2010; 26(2):286-298.

Artigo apresentado em 10/08/2010 Aprovado em 14/10/2010

Versão final apresentada em 10/02/2011 